\title{
Illuminating the dark zone
}

\begin{abstract}
In a recent paper published in Cell, Dustin, Nussenzweig and colleagues illuminate the dynamics of germinal centre B cell migration and show that T cell help is the limiting factor for the selection of high-affinity B cells.

Although much research has focused on defining the mechanisms of $\mathrm{B}$ cell selection in germinal centres and the importance of migration between germinal centre dark and light zones, a clear picture of the dynamics of B cell selection in these structures is lacking. To address this issue, the authors generated transgenic mice that expressed photoactivatable green fluorescent protein (PA-GFP) in their haematopoietic cells. These cells can be photoactivated with great precision
\end{abstract}

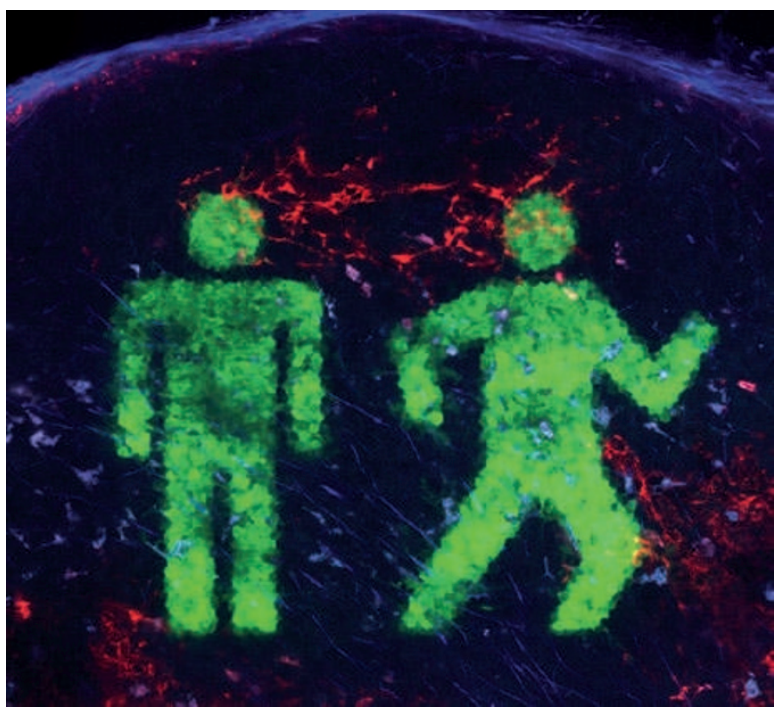

B cells expressing photoactivatable green fluorescent protein can be photoactivated with high spatial precision in germinal centres. Follicular dendritic cells in the germinal centre light zone are labelled with an antigen-conjugated red fluorescent protein and collagen is in blue. Image courtesy of G. D. Victora and M. Nussenzweig, The Rockefeller University, New York, USA. (close to one cell diameter) in these mice and can be analysed by multiphoton laser scanning microscopy and flow cytometry. This method allows for the precise labelling, tracking and molecular analysis of cells within living tissues. The authors then generated antigen-specific germinal centres in which transferred antigenspecific PA-GFP-expressing B cells could be specifically photoactivated in either the dark zone or the light zone. The light zone was identified by injecting an antigen-conjugated red fluorescent protein. In antigenimmunized mice this forms immune complexes that bind follicular dendritic cells (FDCs), which are confined to the light zone.

The authors found that dark zone B cells were CXCR $4{ }^{\text {hi }} \mathrm{CD} 83^{\text {low }} \mathrm{CD} 86^{\text {low }}$ and expressed genes associated with cell division and somatic hypermutation at high levels, whereas light zone B cells were CXCR $4^{\text {low }} \mathrm{CD} 83^{\text {hi }} \mathrm{CD} 86^{\text {hi }}$ and expressed activation markers (associated with antigen encounter and $\mathrm{T}$ cell help) and apoptosis regulators. Analysis of B cell migration in germinal centres in the popliteal lymph nodes of living mice showed that photoactivated dark zone B cells migrated rapidly to the light zone, with up to $50 \%$ of cells migrating to the light zone in 4 hours. By contrast, migration from the light zone to the dark zone was slow, with only $15 \%$ of cells making the transition in 6 hours. These observations are consistent with the model in which the dark zone acts as a source of proliferating $B$ cells with mutated B cell receptors (BCRs) that migrate to and undergo selection in the light zone. B cells in the light zone must compete for a limiting factor that promotes their return to the dark zone for further rounds of proliferation.

But what is the limiting factor? It has been proposed that affinity-based selection of B cells may be driven by BCR cross-linking by antigen that is deposited in immune complexes on FDCs and/or by help from follicular helper $\mathrm{T}\left(\mathrm{T}_{\mathrm{FH}}\right)$ cells following antigen uptake and presentation by high-affinity B cells. The authors devised a model to address the exact mechanism of B cell selection, and found that targeting $\mathrm{T}_{\mathrm{FH}}$ cell help to a subpopulation of $\mathrm{B}$ cells resulted in migration of these $B$ cells from the light zone to the dark zone. They also showed that $\mathrm{T}_{\mathrm{FH}}$ cell help was required for clonal expansion and affinity maturation of these B cells. BCR cross-linking was necessary but not sufficient for affinity maturation, indicating that $\mathrm{T}_{\mathrm{FH}}$ cell help is the limiting factor for $\mathrm{B}$ cell selection in germinal centres.

So, using a unique method that combines PA-GFP expression in $B$ cells with multiphoton microscopy and flow cytometry, this study defines specific mechanisms that govern B cell selection in germinal centres.

Olive Leavy

ORIGINAL RESEARCH PAPER Victora, G. D. et al. Germinal center dynamics revealed by multiphoton microscopy with a photoactivatable fluorescent reporter. Cell 143, 592-605 (2010) FURTHER READING Cyster, J. G. Shining a light on germinal center B cells. Cell 143, 503-505 (2010) 